\title{
Accuracy of fine needle aspiration cytology in the diagnosis of palpable head and neck masses in a tertiary health care center
}

\author{
G. G. Swamy ${ }^{1}$, A. Singh ${ }^{2}$, J.M. Ahuja ${ }^{3}$, N. Satyanarayana ${ }^{4}$ \\ ${ }^{1}$ Associate Professor, ${ }^{2}$ Lecturer, ${ }^{3}$ Professor Dept. of Pathology, ${ }^{4}$ Asst. Professor, Dept.of Anatomy, College of Medical Sciences, \\ Bharatpur, Chitwan, Nepal
}

\begin{abstract}
Palpable masses in head and neck are a common clinical finding, affecting all age groups. These lumps may be extremely worrying for both physician and patient, as a wide variety of pathological conditions. Accurate cytological analysis has played a major role in evaluation and planning for surgery. We attempted to evaluate the role of Fine Needle Aspiration Cytology (FNAC) in diagnosing lesions of the head and neck region and to review the diversity of lesions in the patients attending the hospital. The study was conducted retrospectively in the department of pathology at College of Medical Sciences- Teaching Hospital, Bharatpur, Nepal a tertiary health care centre. The target population comprised patients presenting with palpable masses at head and neck region during the period of February 2007 to December 2009. The accuracy of FNAC was verified by histological examination in this final study group of $(n=125)$ patients. In these $(n=125)$ patients, twenty five were males and hundred were females. Thyroid gland $(60 \%)$ was the commonest site aspirated, followed by lymph node (20\%), salivary gland (16\%) and soft tissue lesions (4\%). In our study the sensitivity was $87.5 \%$, the specificity was $100 \%$, the positive predictive value was $100 \%$, the negative predictive value was $98.26 \%$ and false negatives were $12.5 \%$. We concluded that FNAC is a safe, cost-effective, sensitive and specific technique in the initial evaluation of head and neck masses. A correct cytological diagnosis can be achieved in a majority of cases, avoiding the need for surgical interventions.
\end{abstract}

Key words: FNAC, thyroid, lymph node, salivary gland, soft tissue.

\section{Introduction}

Head and neck masses often pose a challenging diagnostic problem to the clinician. Malignancy remains an important differential diagnosis and neck mass is often the first or the only symptom of this disease. Although surgical biopsy is the commonest method of tissue diagnosis, Fine Correspondence: G. G. Swamy

Email: drgangadhar@hotmail.com
Needle Aspiration (FNAC) is in practice since long time. Fine Needle Aspiration Cytology for evaluation of a neck mass was first used by Kun in 1847, but failed to gain recognition and importance in those times. ${ }^{1}$ Later, in 1930, Martin and Ellis rediscovered this technique in the diagnosis of various organ lesions. ${ }^{2}$ Over a period of years, FNA biopsy has become established as an accurate, safe, and minimally invasive technique and one of the 
Journal of College of Medical Sciences-Nepal,2010,Vol-6,No-4

preferred first-line diagnostic tools. Performed most often for palpable swellings (commonly lymph node, thyroid and salivary gland) in the region of the head and neck, it encompasses a wide range of differential diagnoses, ranging from inflammatory and infective lesions to neoplastic lesions. Accurate cytological analysis has played a major role in evaluation and planning for surgery of head and neck lesions.

We aimed to evaluate the role of Fine Needle Aspiration in diagnosing lesions of the head and neck region and to review the diversity of lesions in the patients presenting at tertiary health care hospital. For these lesions, the result of cytology were compared with those of histopathology, wherever possible. This method has become popular as a diagnostic step in the evaluation of a head and neck mass. ${ }^{3}$

\section{Materials and methods}

The study was conducted retrospectively in the department of pathology at College of Medical Sciences- Teaching Hospital, Bharatpur, Nepal, a tertiary health care centre. The target population comprised patients presenting with palpable masses at head and neck region during the period of February 2007 to December 2009, who underwent Fine Needle Aspiration during the study period. Detailed information regarding the patient, lesion, diagnosis, and any histopathology was recorded from the data. In all cases; Fine needle aspiration was done using a 23 -gauge needle fitted to a $10 \mathrm{ml}$ disposable syringe. The aspirated material was smeared on glass slides, immediately fixed in $95 \%$ ethanol and stained with Hematoxylin and Eosin, and Papaniculaou stain. In all cases, where there was suspicion of tuberculosis or where purulent material was aspirated, modified Zeihl-Neelsen staining was performed to look for the presence of acid-fast bacilli. Surgically excised specimens routinely processed and stained with Hematoxylin and Eosin. These histopathology slides were reviewed and compared with cytological reports for any discrepancy in diagnosis.

\section{Results}

Among the 366 patients, 16 were excluded from the study as the smears were unsatisfactory. The distribution of the 350 cases is given in Table.1. The accuracy of FNAC was verified by histological examination in the 125 patients. Among the 125 patients, 25 were males and 100 were females (M: F ratio $=1: 3)$. Thyroid gland $(60 \%)$ was the commonest site aspirated, followed by lymph node (20\%), salivary gland (16\%) and soft tissue lesions (4\%). (Table.2)

A total of 75 patients with thyroid swelling were identified: 6 (8\%) were male and $69(92 \%)$ were females. Age of the patients ranged from 14 to 70 years. Commonest presentation was neck swelling in $60(80 \%)$ of the patients. Duration of complaints ranged from six days to twenty years and mean duration was 1.7 years. FNAC results revealed 39 (52\%) cases as colloid nodular goiter (figure.1), $12(16 \%)$ as follicular neoplasm (figure.2), $9(12 \%)$ as papillary carcinoma, $6(8 \%)$ as hurthle cell lesions, $6(8 \%)$ as benign cystic lesions, and $3(4 \%)$ cases as suspected of malignancy. Histopathological examination of excised specimens showed $42(56 \%)$ cases as colloid 
G.G. Swamy et al, Accuracy of fine needle aspiration cytology......

nodular goiter, $12(16 \%)$ as follicular adenoma, 12 $(16 \%)$ as papillary carcinoma, $3(4 \%)$ as hurthle cell adenoma, 3 (4\%) as hurthle cell changes with capsular invasion and, 3 (4\%) as hashimoto's thyroiditis. Comparison of FNAC with histopathological findings was performed. 45 cases were diagnosed as colloid nodular goiter and benign cystic lesions by FNAC. Thirty nine of these cases were found to be non neoplastic lesions, three as papillary carcinoma and three as follicular adenoma in histopathological examination. Thirty cases were diagnosed as neoplastic lesions (follicular neoplasm, hurthle cell lesions, papillary carcinoma, and suspected malignancy) by FNAC. Three of these cases were non neoplastic lesions, 12 were benign neoplastic lesions, 12 were carcinoma and 3 cases of suspected malignancy were diagnosed as hashimoto's thyroiditis on histopathological examination. A total of 15 cases of solitary thyroid nodules were diagnosed as having malignant and the most common malignant lesion detected was papillary carcinoma, 12 out of 15 (80\%). Statistical analysis of neoplastic lesions showed sensitivity, specificity, accuracy, false positive rate, false negative rate, positive predictive value, and negative predictive value of FNAC to be $80 \%$, $86.6 \%, 84 \%, 13.3 \%, 20 \%, 80 \%$, and $86.6 \%$ respectively whereas statistical analysis of carcinomatous lesions showed sensitivity, specificity, accuracy, false positive rate, false negative rate, positive predictive value, and negative predictive value of FNAC to be $80 \%, 95 \%$, $92 \%, 5 \%, 20 \%, 80 \%$, and $95 \%$.

Among twenty five patients with lymph node swelling comprised of 5 males and 20 females and their ages ranged from 4-60 years. Reactive lymphadenopathy was the commonest cause of lymphadenopathy, followed by tuberculous, granulomatous and metastatic lymphadenopathy. The present study did not make any attempt to categorize the type of Reactive lymphadenopathy. All ten cases of tuberculous lymphadenopathy showed moderately cellular smears. There were epithelioid granulomas (figure.3) in seven cases and caseation necrosis in three cases. The special stain for acid fast bacilli was positive in four cases. One case of suppurative lymphadenitis which was confirmed by histological diagnosis. Four cases of granulomatous lymphadenitis were correctly diagnosed by cytology. The smears showed focal collections of epithelioid cells in association with reactive lymphoid cells. The special stain for AFB showed negative results. One case of metastatic carcinoma showed tumor cells in sheets and they were dispersed singly. These cells exhibited pleomorphism and had hyperchromatic nuclei with dense cytoplasm in a necrotic background. This was subsequently confirmed by histopathology.

Twenty cases of salivary gland swellings were included in our study. Sex distribution showed 9 (45\%) male patients and 11 (55\%) female patients. In this series of Fine needle aspiration, 8 cases (40\%) were benign neoplasms, 3 cases $(15 \%)$ were malignant neoplasms, one case $(5 \%)$ was non neoplastic cyst and 8 cases (40\%) were inflammatory lesions. Malignant lesions were common in 45-60 yrs age group with a male predominance. Adenoid cystic carcinoma was found in three cases followed by one each of acinic cell carcinoma and mucoepidermoid carcinoma. 
Journal of College of Medical Sciences-Nepal,2010, Vol-6,No-4

Benign lesions were common in 30-40 yrs age group with a female predominance. The commonest benign lesion was pleomorphic adenoma (30\%) followed by warthin's tumour (5\%) and vascular tumors (5\%). Histopathology was correlated with cytology in 11 cases out of which 15 cases. There were no false positive reports but false negative result was seen in 4 cases. Sensitivity and specificity were found to be $90 \%$ and $100 \%$ respectively.

Table 1: Distribution of head and neck cases.

\begin{tabular}{lcc}
\hline Location & Number of cases & Percentage \\
\hline Thyroid & 175 & 50 \\
Lymph node & 100 & 28.57 \\
Salivary gland & 50 & 14.28 \\
Soft tissue & 25 & 7.14 \\
\hline
\end{tabular}

Table 2: Distribution of organs in fine needle aspiration.

\begin{tabular}{lc}
\hline Site & Percentage \\
\hline Thyroid & $60 \%$ \\
Lymph node & $20 \%$ \\
Salivary gland & $16 \%$ \\
Soft tissue & $4 \%$ \\
\hline
\end{tabular}

Patients with soft tissue lesions comprised of five males and their ages ranged from 16-65 years. Three cases of Lipoma, One case each of hamartoma and spindle cell tumour were cytologically diagnosed and confirmed by histopathology.

In the 125 cases, the sensitivity was $87.5 \%$, the specificity was $100 \%$, the positive predictive value was $100 \%$, the negative predictive value was $98.26 \%$ and false negatives were $12.5 \%$.

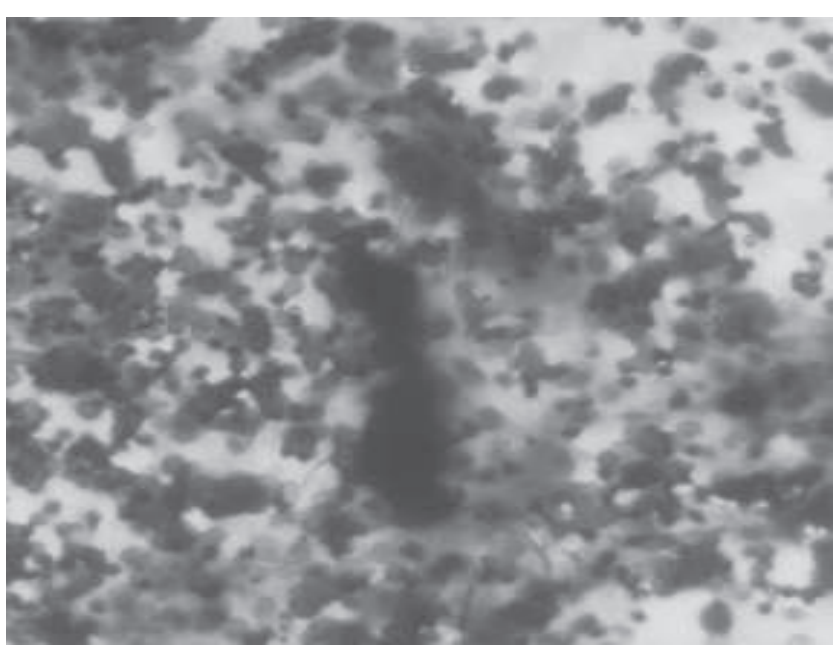

Fig.1: Colloid goitre on FNAC H\&E 100x

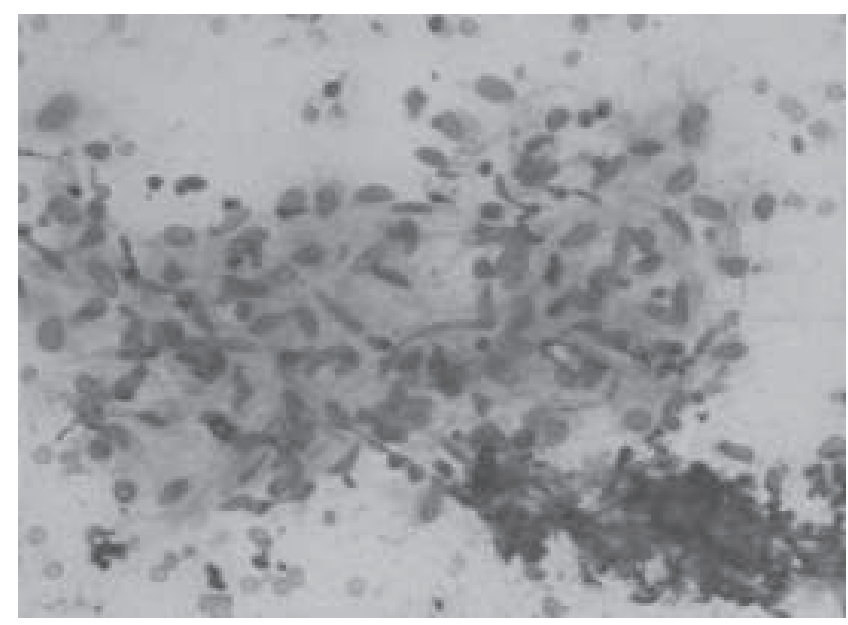

Fig.3: FNAC of Tuberculous Lymphadenitis H\&E 400X

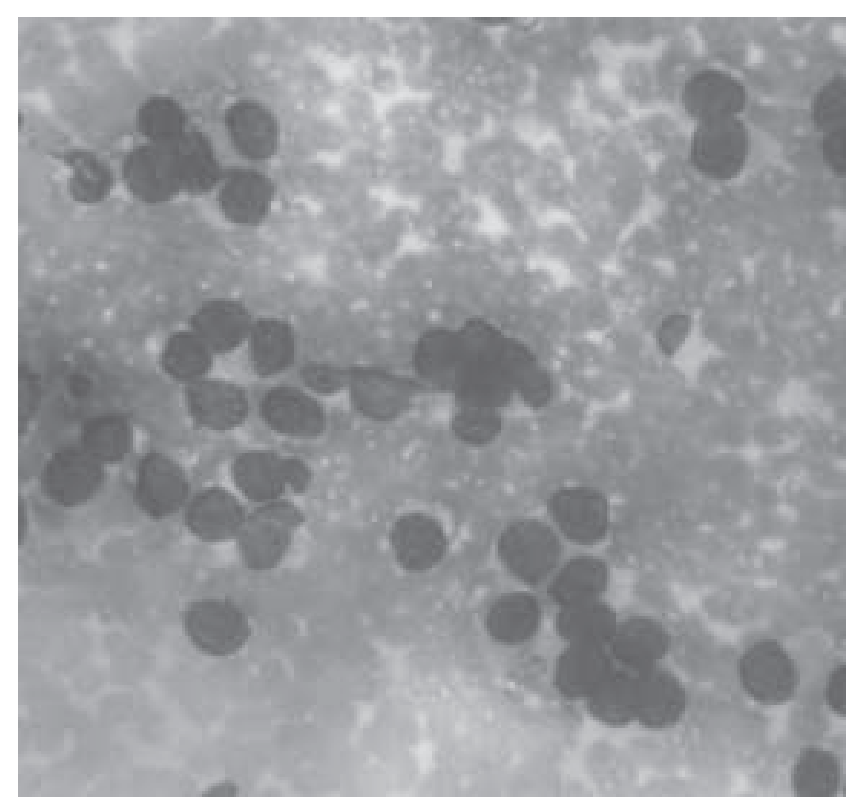

Fig.2: FNAC of Follicular Neoplasm of Thyroid H\&E 400X 
G.G. Swamy et al, Accuracy of fine needle aspiration cytology.

\section{Discussion}

In present study, thyroid swelling was 4-9 times more common in females as compared to males. ${ }^{4,5}$ Our study showed that solitary thyroid nodules were 11 times more common in females than males. The false negative rate was $20 \%$ in cases of neoplastic lesions. It constitutes a serious limitation of this technique since these malignant lesions would go untreated. The incidence of false negative results is as low as $1 \%$ to as high as $30 \% .^{6,7}$ The false positive rate was $13.3 \%$ for neoplastic lesions but none of these lesions were malignant. Compared the results of present study with various previous studies. The methods used for the calculation of sensitivity, specificity, accuracy, positive predictive value, and negative predictive value were similar to previous studies. ${ }^{8,9}$ Sensitivity and accuracy of FNAC for detection of neoplasm were $80 \%$ and $84 \%$, respectively, whereas they were $76 \%$ and $69 \%$, respectively, in a study by Cusick et al. ${ }^{9}$ The sensitivity, specificity, and accuracy of FNAC for, thyroid swellings were $80 \%, 86.6 \%$, and $84 \%$, respectively, in our study whereas sensitivity, specificity, and accuracy of FNAC were $93.5 \%$, $75 \%$, and $79.6 \%$, respectively, in a study by Bouvet et $\mathrm{al}^{5}$ and $79 \%, 98.5 \%$, and $87 \%$, respectively, in a study by Kessler et al. ${ }^{10}$ In our study 15 cases were found to be malignant on histopathological examination (12 papillary carcinoma and 3 hurthle cell lesions). It is to be stressed that all cases of papillary carcinoma diagnosed by FNAC were papillary carcinoma on histopathological examination also. This is in accordance with previous studies. ${ }^{4,10}$ The incidence of malignancy in this study was $20 \%$ which is in accordance with study by Dorairajan and Jayashree. ${ }^{4}$ The incidence of malignancy can be as high as $43.6 \% .^{5}$ The incidence of papillary carcinoma in the present study was $80 \%$. In the literature, incidence of papillary carcinoma varies from $50 \%$ to $80 \% .^{4,5,12}$ Brooks et $\mathrm{al}^{11}$ found that preoperative FNAC had no direct impact on the selection of the surgical procedure.

FNAC diagnostic accuracy rate in tuberculous lymphadenitis is as high as $90-100 \%$. FNAC coupled with ZN staining for AFB is a very useful diagnostic tool in the diagnosis of tuberculous lymphadenitis. There are problems in arriving at a definitive diagnosis in certain cases of Tuberculous lymphadenitis, when the aspirate shows a polymorphous picture with occasional epithelioid cells, with an absence of Langhan's giant cells or caseous necrosis, making it necessary to resort to excisional biopsy for a definitive diagnosis. This is particularly true in children, in whom a similar picture may be seen in cases of reactive hyperplasia due to viral or toxoplasma infection, since the mere presence of the epithelioid cells is not diagnostic of any specific condition. ${ }^{13}$ Eight cases of Reactive lymphadenopathy diagnosed cytologically, confirmed by histopathological studies. The slides were reviewed to look for the typical cytomorphological features. One case diagnosed by cytology as reactive lymphadenopathy but, it was diagnosed by tuberculous lymphadenopathy by histopathological studies.

Salivary gland swellings can present in a variety of locations, depending on the salivary gland affected. A swelling that arises in the parotid or submandibular gland usually presents as an upper neck mass. A swelling of a minor salivary gland or sublingual gland typically presents as an intra-oral swelling. However, minor salivary gland tumors can sometimes occur in other areas of the upper aero digestive tract including the nasal cavity, paranasal sinuses and larynx. Fine-needle aspiration 
cytology of the salivary gland is a commonly accepted, sensitive and specific technique in the diagnosis of both neoplastic and non-neoplastic lesions of the salivary gland. In this study the commonest benign lesion was plemorphic adenoma (30\%) followed by warthin's tumour $(5 \%)$ and vascular tumour (5\%). S.Elagoz et $\mathrm{al}^{14}$ found pleomorphic adenoma (45\%) followed by warthin's tumour (9\%). This study shows adenoid cystic carcinoma is the commonest malignancy followed by acinic cell carcinoma. C.J.R. Stewart et al ${ }^{15}$ also found that adenoid cystic carcinoma (2 cases) is the commonest malignancy. The most common age group of salivary gland swellings was $21-40$ years $(52.5 \%)$ followed by $41-60 \mathrm{yrs}(35 \%)$ with a female predominance. The occurrence of salivary gland tumour increases with age $\mathrm{H}$ Keun et $\mathrm{al}^{16}$ found in another study. Non-neoplastic lesions of the parotid are not uncommon. In our study inflammatory lesions \& non neoplastic cysts were diagnosed. Z Baren et al ${ }^{17}$ found 13 inflammatory lesions and 5 non-neoplastic cysts. Arshad et al ${ }^{18}$ found 20 inflammatory lesions and 10 non-neoplastic cysts. Differential diagnosis of non neoplastic salivary lesions is required to exclude benign and malignant salivary tumors. Fine Needle Aspiration Cytology (FNAC) of a parotid mass, to distinguish neoplastic lesion from inflammatory mass is very useful. ${ }^{19}$ Usually tumors are painless, but adenoid cystic carcinomas are associated with pain due to perineural invasion. Hence it is diagnosed as an inflammatory lesion by the clinicians. Differentiation on clinical grounds alone is not accurate. ${ }^{19}$ There were no false positive reports but false negative results were seen in four cases. Sensitivity and specificity were $90 \%$ and $100 \%$ respectively.

One case of Lipoma was correctly diagnosed by cytology. One case of Hamartoma diagnosed by cytology proved to be Capillary haemangioma after histological studies. One case of Spindle cell tumour which was suspected to be malignant turned out to be malignant peripheral nerve sheath tumour. Fine Needle Aspiration usually faces no problem in distinguishing high grade soft tissue sarcomas from benign lesions. However, borderline and low grade lesions are susceptible to be missed. Accurate typing and grading of the tumour is not possible in many cases by fine needle aspiration alone. Almost all studies on soft tissue tumours have reported this limitation of fine needle aspiration. ${ }^{20}$

On comparing the results of the present series with other workers, it can be said that the results of this study are favorable with those published in literature and are fairly accurate.

\section{Conclusion}

We conclude that FNA biopsy is a simple and rapid procedure which can be carried out as an out patient procedure with minimal problems. Owing to its accurate diagnostic potential in benign and malignant lesions, we recommend FNA biopsy as the first-line investigation in diagnosing head and neck swellings.

\section{Acknowledgement}

We wish to thank all clinical Departments for sending the patients for FNAC and specimens for histopathological examination to our Dept and also thank to Dr. Merina, Dr. Sushna Maharjan, Dr. Rajina Sahi, Dr. Agya Shrestha, Residents of Pathology, Mr. P.K. Mishra, Mr. Prakash Lab. Technicians, Dept. of Pathology for their technical help. Special thanks to Mrs. Shobha Neupane for her help in preparation of this manuscript. 
G.G. Swamy et al, Accuracy of fine needle aspiration cytology.

\section{References}

1. J.E. Russ, E.F. Sacnion, A.M. Christ. Aspiration cytology of head and neck masses. Am J Surg. 1978;136:342-7.

2. G. Mahbod, F. Koasri, M.A. Tafreshi. Fine needle aspiration cytology in diagnosing non thyroidal neck masses. Acta Med Iran. 2002;40:49-51.

3. V.Tilak, A.V. Dhaded, R. Jain. Fine needle aspiration cytology of head and neck masses. Indian JPathol Microbiol 2002;45(1):23-30.

4. N. Dorairajan, N. Jayashree. "Solitary nodule of the thyroid and the role of fine needle aspiration cytology in diagnosis. J Ind Med Ass 1996;94(2):50-2.

5. M.Bouvet, J.I. Feldman, G.N. Gill et al. Surgical management of the thyroid nodule: patient selection based on the results of fine-needle aspiration cytology. Laryngoscope 1992;102(12):1353-6.

6. R.C. Hamaker, M.I.Singer, R.V. DeRossi, et al. Role of needle biopsy in thyroid nodules. Archives of Otolaryngology 1983;109(4):225-8.

7. M.Radetic, Z.Kralj, I.Padovan. Reliability of aspiration biopsy in thyroid nodes: study of 2190 operated patients. Tumori 1984;70 (3):271-6.

8. H.M.Al-Sayer, Z.H. Krukowski,V.M.Williams, et al. Fine needle aspiration cytology in isolated thyroid swellings: a prospective two year evaluation. Brit Med J 1985; 290:1490-2

9. E.L.Cusick, C.A.MacIntosh, Z.H.Krukowski et al. Management of isolated thyroid swelling: a prospective six year study of fine needle aspiration cytology in diagnosis. Brit Med J 1990;301:318-21.

10. A.Kessler, H.Gavriel, S.Zahav, et al. Accuracy and consistency of fine-needle aspiration biopsy in the diagnosis and management of solitary thyroid nodules. Israel Med Ass J 2005;7(6):371-3.
11. A.D.Brooks, A.R.Shaha,W.D.Mornay. Role of fine needle aspiration biopsy and frozen section analysis in the surgical management of thyroid tumors . Annals of Surgical Oncology 2001;8(2):92-100.

12. R.J. DeVos, T.N. Cappel, N.D. Bouvy et al. Fine needle aspiration cytology of thyroid nodules: how accurate is it and what are the causes of discrepant cases. Cytopath $200 ; 12$ (6) :399-405.

13. A.K.Gupta, M. Nayar, M.Chandra. Critical appraisal of fine needle aspiration cytology in tuberculous lymphadenitis. Acta cytol 1992;36(3):391-4.

14. S.Elagoz, M.Gulluoglu, D.Y.Imazbayhan, et al. The value of fine-needle aspiration cytology in salivary gland lesions. $J$ Otorhinolaryngol Relat 2007;69(1):51-6.

15. C.J.R. Stewart, K.MacKenzie, G.W. McGarry. et al. fineneedle aspiration cytology of salivary gland: A review of 341 cases. Diag Cytopathol 2000;22(3):139-46.

16. L.Keun-Hwa, C.Myung-Je, Y.Yoshio. FNAC diagnosis of salivary gland swellings. J Clin Pathol 1994;87:231-6.

17. Z.Baren, P.Schar, M.A.Hotz, et al. Value of fine needle aspiration cytology of parotid gland masses. Laryngoscope 2001;111:1989-92.

18. A.R.Arshad. Parotid swellings:report of 110 nsecutive cases. Med J Malaysia. 1998; 53:417- 22.

19. A.R.Shaha, C.Webber, T.DiMaio. Needle aspiration biopsy in salivary gland lesions. Am J Surg 1990;160:373.

20. S. Kumara, N. Chowdhury. Accuracy, limitations and pitfalls in the diagnosis of soft tissue tumours by fine needle aspiration cytology. Indian J Pathol Microbiol. 2007;50 (1):42-5. 